\title{
Social Behaviour Inspired Optimization Algorithm: An Approach for Solving Complex Optimization Problems

\author{
${ }^{1,2}$ Department of CSE, G H Raisoni College of Engineering, Nagpur, India
} \\ ${ }^{* 1}$ Priya Chandel, ${ }^{2}$ Prashant Borkar \\ *Email: priyachandel.263@gmail.com
}

\section{Received: 09 ${ }^{\text {th }}$ July 2018, Accepted: $14^{\text {th }}$ August 2018, Published: 31 ${ }^{\text {st }}$ August 2018}

\begin{abstract}
Inspired from the human behavior an advanced Social Behavior Inspired Optimization rule is given in this research work. This rule works in two phases inspired from human behavior in phase one candidate solution is improved considering the good behavior and by avoiding the bad behavior and in second phase solution is improved by knowledge transfer among the candidates. This algorithmic rule does not depend on any algorithmspecific controlling parameters and needs only common controlling parameters. The execution of Social behavior impressed optimization algorithm is tested on standard benchmark functions having different characteristics and compared with traditional optimization algorithms such as GA, DE, PSO, ABC, Jaya and also with TLBO algorithm.
\end{abstract}

Keywords: Social Behavior, Constrained Optimization, Unconstrained Optimization, Benchmark Function.

\section{Introduction}

An extensive variety of optimization rules are there in swarm and evolutionary computation like PSO, GA, ACO, HS, ABC, etc. are a few of them [16]. Swarm intelligence models the collective behavior of animals or swarms of insects and has attracted the attention of researchers.

Optimization is the act of getting the most effective result underneath given circumstances. In design, construction, and maintenance of engineering system, we've to require several technological and social control choices at many stages. The last goal of all such choices is either to reduce the efforts needed or to maximize the specified advantages. Since the efforts required or profit desired in any useful circumstance is frequently communicated as an objective function of some design variables. Optimization process can be viewed as the way toward discovering the conditions that can help in finding the maximum or minimum value of an objective function. Besides the evolutionary techniques and swarm insight based calculations, deal with the principles of various phenomenon such as HS algorithmic rule, GSA, BBO, GEM, and so on [4]. All the biological process and swarm knowledge basically based calculations require normal dominant parameters such as population size, a variety of generations, size of elite, etc. Besides the basic administration parameters, totally unique calculations require their own particular calculation particular administration parameters. For instance, GA, PSO, ABC, HS depends on different algorithmic-specific parameters and therefore need the variety of improvisations. Likewise, the contrary calculations like ES, DE, ACO, BFO, EP, AIA, SFL, and so on need the standardization of different calculation particular parameters. The right standardization of the algorithmic rule specific parameters could be a terribly difficult issue that affects the ability of most of the mentioned optimization techniques. The improper standardization of algorithm specific controlling parameters may increase processing effort or lead to the native best answer.

Assuming this reality, presented within the teaching learning primarily based optimization algorithmic rule that doesn't need standardization of any algorithm specific controlling parameters. The TLBO algorithmic rule needs solely common dominant parameters for its operating. Keeping seeable of the success of the TLBO and Jaya algorithmic rule, another algorithmic rule specific parameter-less algorithm is planned. The planned algorithmic rule works in two phases and it needs solely the common control parameters for its operating. The social behavior inspired optimization technique is described in next section.

\section{Proposed Algorithm}

Figure1. Shows the flow diagram of the Social Behavior Inspired Optimization Algorithm. Inspired from human behavior in phase1, the algorithm continually tries to get nearer to success (by considering the good behavior) and tries to avoid failure (by neglecting the bad behavior). By using following equation,

$\mathrm{G}_{\mathrm{j}, \mathrm{k}, \mathrm{i}}^{\prime}=\mathrm{G}_{\mathrm{j}, \mathrm{k}, \mathrm{i}}+\mathrm{r} 1_{1 \mathrm{j}, \mathrm{i}}\left(\mathrm{G}_{\mathrm{j}, \text { best, } \mathrm{i}}-\left|\mathrm{G}_{\mathrm{j}, \mathrm{k}, \mathrm{i}}\right|\right)-\mathrm{r} 2_{\mathrm{j}, \mathrm{i}}\left(\mathrm{G}_{\mathrm{j}, \mathrm{worst}, \mathrm{i}}\left|\mathrm{G}_{\mathrm{j}, \mathrm{k}, \mathrm{i}}\right|\right)$

In phase2, candidates increase their result by knowledge transfer by using following equation. A candidate interacts randomly with any other candidate in population for improving his or her own solution if the opposite candidate has additional data than him.

$$
\begin{aligned}
& \text { Gtinew } \quad=\quad\left\{G t_{i o l d}+\text { rand }_{1}\left(G t_{i}-G t_{j}\right)\right. \\
& f\left(G t_{i}\right)<f\left(G t_{j}\right) \text { Gtiold }+ \text { rand }\left(G t_{j}-G t_{i}\right) \text { if } f\left(G t_{j}\right)<f\left(G t_{i}\right)
\end{aligned} \text { if }
$$

\section{Social Behavior Inspired Optimization Algorithm} The important steps in Social Behavior Inspired optimization technique are as given below, Initialize the population 
1: repeat

2: Estimate the fitness of every candidate in population

3: Phase one: Update the candidate solution based on best behavior and worst behavior

4: Phase two: Modify the candidate based on knowledge transfer and update the solution

5: until requirements are met

Demonstration of the working of Social Behavior Inspired Optimization algorithm

To demonstrate the operating of Social Behavior Inspired Optimization technique, an unconstrained benchmark objective function sphere is used. The objective of sphere objective function is to find values for xi that helps in minimizing value of benchmark function sphere.

$$
\begin{aligned}
& \min f(x)=\sum_{i=1}^{n} x_{i}{ }^{2} \\
& \text { Subject to } \\
& -100 \leq x_{i} \leq 100
\end{aligned}
$$

As it is minimization problem, known solution for sphere benchmark function will be considered as 0 for all values of 0 . To demonstrate operating of Social Behavior Inspired optimization algorithm, initially population size of 5 is considered with $\mathrm{x} 1$ and $\mathrm{x} 2$ as two design variables. Generation of results for sphere target function is indicated in Table 1 from the initial population. For minimization, lowest fitness value of sphere target function indicates good behavior i.e. best solution and highest value indicates bad behavior i.e. worst solution. Results for sphere target function is indicated in Table 1 from the initial population. For minimization, lowest fitness value of sphere target function indicates good behavior i.e. best solution and highest value indicates bad behavior i.e. worst solution.

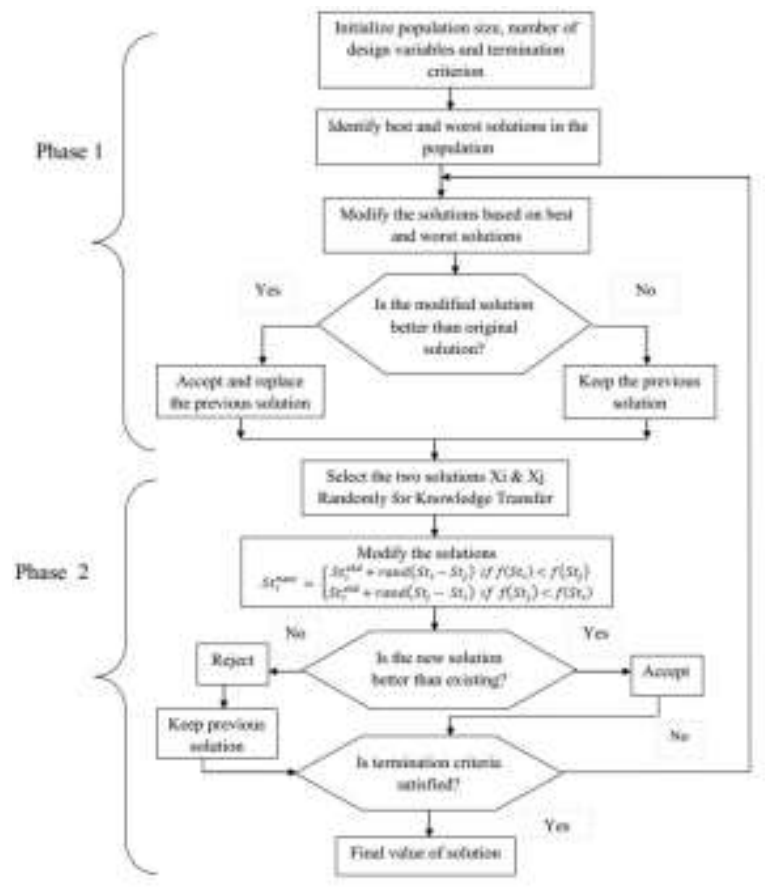

Figure 1: Flow chart of the Social Behavior Inspired Optimization Algorithm

\begin{tabular}{|l|c|c|c|c|}
\hline Candidates & $\mathrm{x} 1$ & $\mathrm{x} 2$ & $(\mathrm{)}$ & Behavior \\
\hline 1 & 62.9447 & 81.1584 & 10548.7211 & \\
\hline 2 & -74.6026 & 82.6752 & 12400.7366 & \\
\hline 3 & 26.4718 & -80.4919 & 7179.7021 & \\
\hline 4 & -44.3004 & 9.3763 & 2050.4404 & Best \\
\hline 5 & 91.5014 & 92.9777 & 17017.3589 & Worst \\
\hline
\end{tabular}

Table 1: Results for Sphere Target Function

Table 2 indicates changed values of the variables $\mathrm{x} 1, \mathrm{x} 2$ and the sphere target function amid phase one of Social Behavior Inspired Optimization algorithm. x1mod and $\mathrm{x} 2 \mathrm{mod}$ are the changed values of design variables and ( )mod represents the altered. Estimations of objective function according to condition (1). x'1 mod x'2 mod are the values acquired in taking care of limit conditions.

\begin{tabular}{|c|c|c|c|c|c|}
\hline Candidates & x1mod & x2mod & x'1mod & x'2mod & ( )mod \\
\hline 1 & 18.3246 & 6.7141 & 18.3246 & 6.7141 & 380.8701 \\
\hline 2 & -172.1561 & 42.3261 & -100.000 & 42.3261 & 11791.4987 \\
\hline 3 & -91.9897 & -127.5712 & -91.9897 & -100.000 & 18462.1049 \\
\hline 4 & -163.6192 & -53.9718 & -100.000 & -53.9718 & 12912.9552 \\
\hline 5 & -9.4173 & 38.1788 & -9.4173 & 38.1788 & 1546.3063 \\
\hline
\end{tabular}

Table 2: Changed Values of the Variables and the Objective Function during Phase One

Table 3 demonstrates the refreshed values of design variables and sphere target function using fitness comparison towards the end of first phase of proposed Optimization algorithm. '1, '2 and '( ) are the new values

\begin{tabular}{|c|c|c|c|}
\hline Candidates & $X^{\prime} 1$ & $X^{\prime} 2$ & '( ) \\
\hline 1 & 18.3246 & 6.7141 & 380.8701 \\
\hline 2 & -100 & 42.3261 & 11791.499 \\
\hline 3 & 26.4718 & -80.4919 & 7179.7021 \\
\hline 4 & -44.3004 & 9.3763 & 2050.4404 \\
\hline 5 & -9.4173 & 38.1788 & 1546.3063 \\
\hline
\end{tabular}
of design variable and sphere target function respectively.

Table 3: Updated Values of the Design Variables and Sphere Objective Function Based on Fitness Comparison at the End of First Phase of Proposed Optimization Algorithm

Table 4 indicates new values of the variables and the target work amid second stage.x1new, x2new and ( ) new will be the changed design values of variables and sphere target work as per condition (2). x'1new x'2new are the values got in the wake of dealing with limit conditions for the unconstrained benchmark work.

\begin{tabular}{|c|c|c|c|c|c|}
\hline Candidates & x1new & x2new & x'1new & x'2new & ()$_{\text {new }}$ \\
\hline 1 & 18.3246 & 6.7141 & 18.3246 & 6.7141 & 380.8701 \\
\hline 2 & -67.2332 & 40.6819 & -67.2332 & 40.6819 & 6175.3201 \\
\hline 3 & 25.6805 & -8.6814 & 25.6805 & -8.6814 & 734.8547 \\
\hline 4 & -0.7867 & 8.5321 & -0.7867 & 8.5321 & 73.4156 \\
\hline 5 & 16.9437 & 37.0950 & 16.9437 & 37.0950 & 1663.1279 \\
\hline
\end{tabular}

Table 4: New Values of the Variables and the Objective Function during Second Phase 
Table 5 demonstrates the refreshed values of the design variables and sphere target function in light of fitness correlation toward the end of stage two. "1 " 2 are the last values of design variable towards the end of first emphasis. Until the point when the termination basis meets these values are given as a contribution to the following emphasis

\begin{tabular}{|c|c|c|c|}
\hline Candidates & X”1 & X”2 & "( ) \\
\hline 1 & 18.3246 & 6.7141 & 380.8701 \\
\hline 2 & -67.2332 & 40.6819 & 6175.3201 \\
\hline 3 & 25.6805 & -8.6814 & 734.8547 \\
\hline 4 & -0.7867 & 8.5321 & 73.4156 \\
\hline 5 & -9.4173 & 38.1788 & 1546.3063 \\
\hline
\end{tabular}

Table 5: Updated Values of the Variables and the Objective Function Based on the Fitness Comparison at the End Second Phase

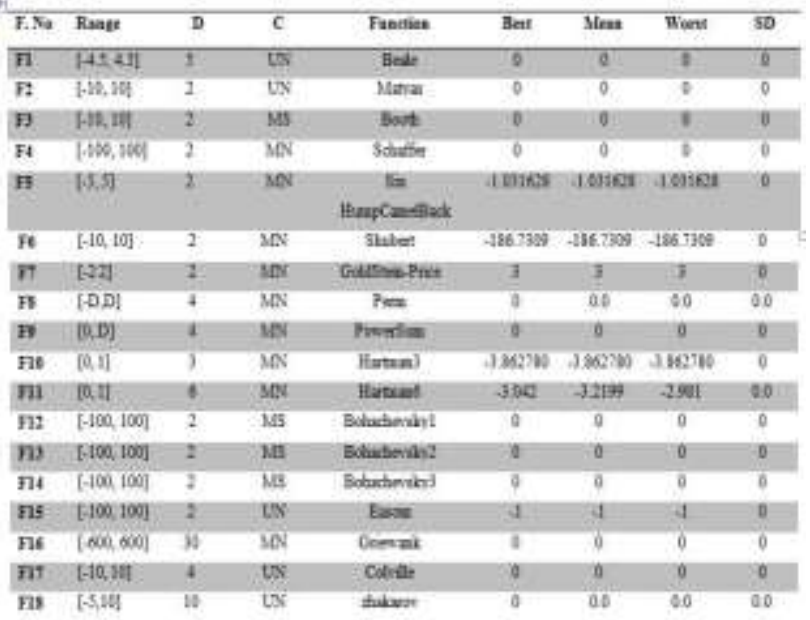

Table 6: Social Behavior Inspired Optimization Algorithm Analogous to each Benchmark Function

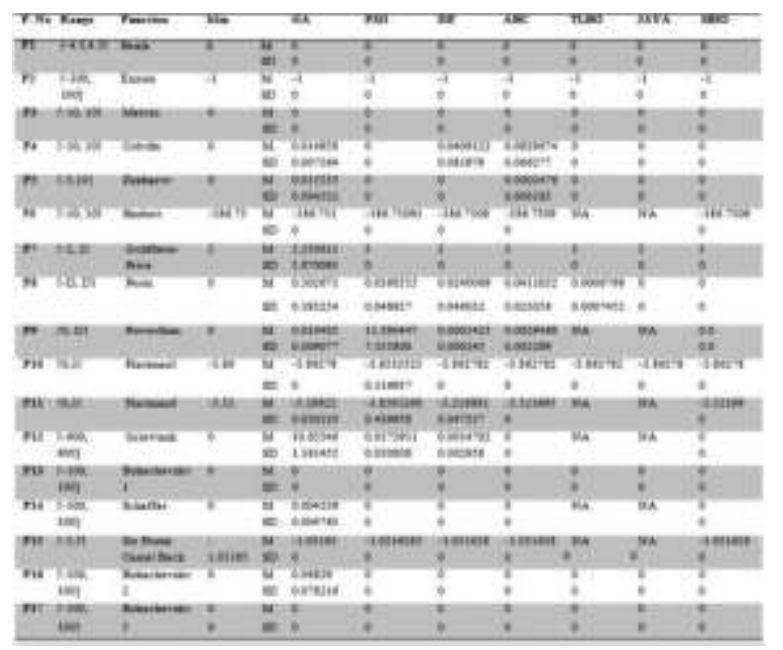

Table 7: Statistical Results of 10 runs Obtained by GA, PSO, DE, ABC, TLBO, Jaya and Social Behavior Inspired Optimization algorithm (SBIO)
D: Dimensions, M: Mean, SD: Standard deviation of the best values, NA: Result not available.

Experiments on Standard Benchmarks Functions

The execution of the proposed Social Behavior Inspired Optimization algorithm is tested on 18 unconstrained benchmark capacities having diverse attributes, for example, Beale, Easom, Matyas, Collvile, Zhakarov, Bohachevsky1, Bohachevsky2, Bohachevsky3, Booth, Scheffer, Six Hump Camel Back, Shubert, GoldStienPrice, Hartman3, Hartman6, Griewank, Perm, PowerSum and all around described in the optimization literature [7]. To analyze the execution of the proposed Social Behavior Inspired Optimization algorithm, the results created by utilizing the Social Behavior Inspired Optimization algorithm are contrasted with the results produced by GA, PSO, DE, ABC, TLBO and Jaya by keeping up the comparative capacity assessments for various algorithms utilized for the examination. While dissecting the result of Social Behavior Inspired Optimization algorithm with other optimization algorithms consistency in the examination is kept up.

The results of Social Behavior Inspired Optimization algorithm comparable to every benchmark work are given in Table 6 as best, most exceedingly terrible, mean arrangement and standard deviation produced 10 independent runs on every benchmark function. The execution of Social Behavior Inspired Optimization algorithm is contrasted with the other surely understood optimization algorithms such as GA, PSO, DE, ABC, TLBO and Jaya and the results are given in Table 7. The outcomes of GA, PSO, DE and ABC are taken from [15], the outcomes of TLBO are listed from [8] and outcomes of Jaya are listed from [7]. It can be seen from Tables 6 and 7 that the proposed Social Behavior Inspired Optimization algorithm has generated better results in terms of "best", "mean" and "worst" solutions of each objective function and "standard deviation". Moreover, it can be noticed that the performance of the Social Behavior Inspired Optimization algorithm is found to be either identical or better than the algorithms taken for comparison for almost all the unconstrained benchmark functions.

\section{Conclusion}

A Social Behavior Inspired Optimization algorithm which is algorithmic parameter less is presented in this research work having two phases. In first stage, the algorithm consistently tries to get closer to success (by considering the great behavior) and tries to stay away from disappointment (by disregarding the terrible behavior) and in second stage, candidates increment their answer by information exchange. The execution of the proposed Social Behavior Inspired Optimization algorithm is tested on 17 unconstrained benchmark capacities having diverse attributes. To assess the 
execution Social Behavior Inspired Optimization algorithm is contrasted with Differential Evolution (DE), Genetic Algorithm (GA), Particle Swarm Optimization (PSO), Artificial Bee Colony Optimization (ABC), Jaya algorithm and Teaching Learning Based Optimization (TLBO). The results have affirmed the effectiveness of the Social Behavior Inspired Optimization algorithm when contrasted with different algorithms.

\section{References}

1. J. Peirong, "A New Genetic Algorithm for Optimization," pp. 1092-1094, 2008.

2. Z. Zhan et al., "Adaptive Particle Swarm Optimization," vol. 39, no. 6, pp. 1362-1381, 2009

3. I. Koohi, "Optimizing Particle Swarm Optimization Algorithm,” pp. 1-5, 2014.

4. Z. Yang, K. Li, A. Foley, and C. Zhang, "A New SelfLearning TLBO Algorithm for RBF Neural Modelling of Batteries in Electric Vehicles," pp. 2685-2691, 2014.

5. C. Paper, N. Lileshwar, H. Raisoni, and N.L. Hatwar, "Teaching Learning Based Optimization Algorithm for Solving Disassembly Sequence Planning Problem Teaching Learning Based Optimization Algorithm for Solving Disassembly Sequence Planning Problem," no. May, pp. $0-5,2016$.

6. N. Lileshwar, H. Raisoni, and N. L. Hatwar, "Disassembly Technique For Software By Disassembly Technique For Software," no. May, 2016.

7. R. V. Rao, "Jaya: A simple and new optimization algorithm for solving constrained and unconstrained optimization problems," vol. 7, pp. 19-34, 2016.

8. R. V. Rao, V. D. Kalyankar, and G. Waghmare, "Parameters optimization of selected casting processes using teaching - learning-based optimization algorithm," Appl. Math. Model., 2014.

9. R. V. Rao and V. Patel, "International Journal of Industrial Engineering Computations," vol. 3, pp. 535- 560, 2012.

10. P. Taylor, R. V. Rao, and V. D. Kalyankar, "Materials and Manufacturing Processes Parameter Optimization of Machining Processes Using a New Optimization Algorithm Parameter Optimization of Machining Processes Using a New Optimization Algorithm," no. March 2014, pp. 37-41, 2012.
11. R. Zou, V. Kalivarapu, E. Winer, J. Oliver, and S. Bhattacharya, "Particle Swarm Optimization-Based Source Seeking," vol.12, no. 3, pp. 865-875, 2015.

12. Y. Yuan and Y. Zhu, "A Hybrid Artificial Bee Colony Optimization Algorithm," pp.492496, 2014.

13. N. Hatwar and S. Nimbhorkar, "IJARCETVOL-5- ISSUE-4-1105-1108 Disassembly Technique for Software by Teaching and Learning Algorithm," no. 4, 2016.

14. J. C. Bansal, P. K. Singh, M. Saraswat, A. Verma, S. S. Jadon, and A. Abraham, "Inertia Weight Strategies in Particle Swarm," pp. 640-647, 2011.

15. D. Karaboga, B. Akay, A comparative study of Artificial Bee Colony algorithm, Applied Mathematics and Computation, 214 (2009) 108-132

16. R. V. Rao, V. Patel, An elitist teachinglearning-based optimization algorithm for solving complex constrained optimization problems. International Journal of Industrial Engineering Computations, 3(4), 535- 560, 2012 